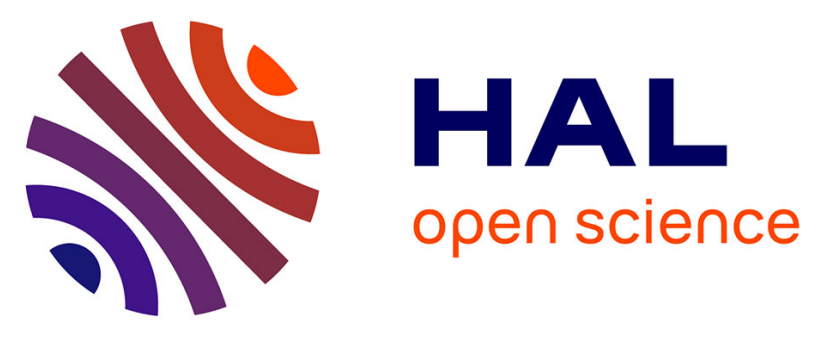

\title{
Density of States Broadening in CH 3 NH 3 PbI 3 Hybrid Perovskites Understood from ab initio Molecular Dynamics Simulations
}

\author{
Liujiang Zhou, Amanda J Neukirch, Dayton Vogel, Dmitri Kilin, Laurent \\ Pedesseau, Marcelo Carignano, Aditya D Mohite, Jacky Even, Claudine \\ Katan, Sergei Tretiak
}

\section{To cite this version:}

Liujiang Zhou, Amanda J Neukirch, Dayton Vogel, Dmitri Kilin, Laurent Pedesseau, et al.. Density of States Broadening in CH 3 NH 3 PbI 3 Hybrid Perovskites Understood from ab initio Molecular Dynamics Simulations. ACS Energy Letters, 2018, 3 (4), pp.787-793. 10.1021/acsenergylett.8b00166 . hal-01724992

\author{
HAL Id: hal-01724992 \\ https://hal.science/hal-01724992
}

Submitted on 6 Jul 2018

HAL is a multi-disciplinary open access archive for the deposit and dissemination of scientific research documents, whether they are published or not. The documents may come from teaching and research institutions in France or abroad, or from public or private research centers.
L'archive ouverte pluridisciplinaire HAL, est destinée au dépôt et à la diffusion de documents scientifiques de niveau recherche, publiés ou non, émanant des établissements d'enseignement et de recherche français ou étrangers, des laboratoires publics ou privés. 


\title{
Density of States Broadening in $\mathrm{CH}_{3} \mathrm{NH}_{3} \mathrm{Pbl}_{3}$
}

\section{Hybrid Perovskites Understood from ab initio}

\section{Molecular Dynamics Simulations}

\author{
Liujiang Zhou, ${ }^{*, \dagger}$ Amanda J. Neukirch, ${ }^{\dagger}$ Dayton J. Vogel, ${ }^{\ddagger}$ Dmitri S. Kilin, ${ }^{\top}$ \\ Laurent Pedesseau, ${ }^{\S}$ Marcelo A. Carignano, $\|$ Aditya D. Mohite, ${ }^{\perp}$ Jacky Even, ${ }^{\S}$ \\ Claudine Katan, ${ }^{*}$ and Sergei Tretiak ${ }^{*} \dagger$ \\ $\dagger$ Theoretical Division, Center for Nonlinear Studies and Center for Integrated \\ Nanotechnologies, Los Alamos National Laboratory, Los Alamos, NM 87545, USA \\ $\ddagger$ Department of Chemistry, University of South Dakota, Vermillion, SD 57069, USA \\ IDepartment of Chemistry and Biochemistry, North Dakota State University, Fargo, ND \\ 58102, USA \\ $\S$ Univ Rennes, INSA Rennes, CNRS, Institut FOTON-UMR 6082, F-35000 Rennes, \\ France
}

\|Qtar Environment and Energy Research Institute, Hamad Bin Khalifa University, Qatar Foundation, P. O. Box 34110, Doha, Qatar

$\perp$ Materials Physics and Application Division, Los Alamos National Laboratory, Los Alamos, NM 87545, USA

\#Univ Rennes, ENSCR, INSA Rennes, CNRS, ISCR (Institut des Sciences Chimiques de Rennes)-UMR 6226, F-35000 Rennes, France

E-mail: ljzhou86@lanl.gov; serg@lanl.gov 


\begin{abstract}
Lead-halide perovskites are the promising materials in optoelectronic devices for their unique properties including direct band gap, strong light absorption, high carrier mobility and low fabrication cost. Here, by using ab inito molecular dynamics and electronic structure calculations, we report a systematic study on the broadening density of states (DOS) deep in the valence bands that has been experimentally observed but absent in static calculations. We quantify the broadening DOS reduction from cubic phase to lower temperature tetragonal and orthorhombic phases and attribute observed effects to the molecular vibrations and the anharmonicity of iodine atoms motion. Specifically, the MA cations' vibrations are strongly linked to the moderate $\mathrm{C}-\mathrm{N}$ stretch and $\mathrm{CH}_{3}$ bend, as well as the strong $\mathrm{CH}_{3}$ and $\mathrm{NH}_{3}^{+}$stretches. These results present a theoretical perspective on the structural dynamics in lead-halide perovskites which may be valuable for future studies toward desired functionalities in perovskite-based optoelectronic devices.
\end{abstract}

\title{
Graphical TOC Entry
}

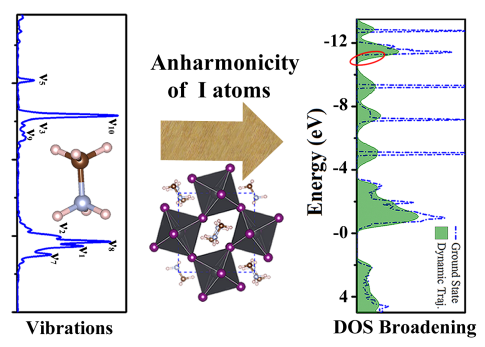


Solution-processed lead-halide perovskites have emerged as a promising optoelectronic technology due to many unique advantages including direct band gap, strong light absorption, high carrier mobility, and low fabrication cost. ${ }^{1}$ For example, the photovoltaic efficiency has jumped spectacularly up to $15-22.1 \%$ for methyl ammonium lead iodine $\left(\mathrm{MAPbI}_{3}\right.$, $\left.\mathrm{MA}=\mathrm{CH}_{3} \mathrm{NH}_{3}\right)$ materials. ${ }^{2-4}$ The underlying mechanisms behind these attractive photoelectronic properties have been widely explored in terms of defects, ${ }^{5}$ ion migration, ${ }^{6-11}$ polaronic effects (including both small ${ }^{12,13}$ and large polarons), ${ }^{14,15}$ ferroelectric order, ${ }^{16-18}$ structural and electronic dimensionality, ${ }^{19}$ etc. Studies of the relationship between the organic cations and device efficiency are rare and are mainly focused on the orientation of the $\mathrm{MA}$ and the induced distortion of the $\mathrm{PbI}_{6}$ octahedral cage as well as direct-indirect band gap transition. ${ }^{20}$ Moreover, the spontaneous generation of free carriers from exciton screening originating from the collective orientational motion of the organic cations has been experimentally confirmed. ${ }^{21}$ Upon change of the organic cation, the carrier self-trapping phenomena, ${ }^{12}$ interband transition properties, and optical absorption strength ${ }^{22}$ are significantly altered. Additionally, due to thermal effects, MA is associated with various molecular vibrational modes and motionally disordered orientations, ${ }^{23}$ which play an important role in the carrier mobility. ${ }^{24}$ The molecular vibrational modes have already been measured by means of Infrared (IR $)^{25}$ and Raman ${ }^{26}$ spectroscopies. Although MA electronic states are not the main contributors around the Fermi level, we expect their vibrations to partly affect material's functionality via enhancing the electronic dimensionality and band transport in devices. ${ }^{19}$

Current theoretical calculations do not match the experimentally measured density of states (DOS) deep within the valence band (VB) of $\mathrm{MAPbI}_{3}$ materials. Calculated DOS has sharp features within the valence band that are in stark contrast to the "flattened" nature of the experimentally measured DOS. ${ }^{27,28}$ Literature has previously stated that due to a higher photo-ionization cross-section, only the $\mathrm{Pb}$ and I ions interact with high energy sources, ${ }^{27}$ making the MA cation a spectator deep within the VB. If this is the case, electronic state 
separation due to spin orbit coupling (SOC), mainly associated with $\mathrm{Pb}$ and $\mathrm{I},{ }^{29,30}$ could be the main factor in DOS broadening. Lack of direct experimental signatures from MA may be attributed to the low photoionization cross sections of light elements, consequently signals of $\mathrm{C}, \mathrm{N}$ and $\mathrm{H}$ atoms are difficult to detect at high photon energies in hard X-ray photoelectron spectroscopy (HAXPES). ${ }^{31}$ Computed valence spectra provide a qualitative agreement with experiment if the DOS peaks are broadened with a $0.3-0.5 \mathrm{eV}$ wide Gaussian function. ${ }^{32,33}$ Such an ad hoc way to account for the effect of molecular motion does not provide insights into the contributing atomic species. However, another hypothesis explaining the difference between the calculated and measured DOS is that the experimental spectra reflect only the average DOS resulting from a convolution of molecular motion and time resolution of spectral equipment. This is expected to recreate experimental observations deep within the VB and thus clarify the good X-ray detection ability of the material. ${ }^{34-36}$ Moreover, the much more flattened and broadened characters of VB dispersion determined by angle resolved photoemission experiments further provides evidence for the feasibility of broadening the DOS by vibrations of MA. ${ }^{37}$ The only way to get a detailed atomistic information on the processes taking place and to quantify the effects of molecular vibrational motions, inorganic lattice anharmonicity, and SOC is to perform molecular dynamics simulations of MA and Cs perovskite systems in different thermodynamic ensembles.

To address the discrepancies between theory and experiment, we consider factors possibly influencing the theoretical DOS. Contributing physical properties, such as SOC and temperature induced distortions (octahedral tilting), ${ }^{38-41}$ may play an important role in the shape and broadening of the DOS. SOC predominantly affects the orbitals of heavy atoms in systems. Previous theoretical calculations stated that the VB just below the Fermi level is derived from $\mathrm{Pb}-6 \mathrm{~s}$ and I-5p states and the conduction band minimum (CBM) is dominated mostly by $\mathrm{Pb}-6 \mathrm{p}$ states, suggesting that SOC mainly influences the CBM in all temperature phases. ${ }^{29,30,42}$ The distortion of the $\mathrm{PbI}_{6}$ octahedral cage induces a direct-indirect band gap transition ${ }^{43}$ and modifies the electronic states around the Fermi level. ${ }^{22}$ Given that the en- 
ergy difference between DOS peaks deep within the VB are on the order of up to $0.5 \mathrm{eV}$, a substantial change in either atomic orientation or electronic distribution must occur in order to justify the wide range of absorption energies experimentally measured. ${ }^{34-36}$ Identification of the atomic species participating in the state energy fluctuations can possibly answer if the broadened DOS primarily originates from molecular motion or re-distribution of electronic states. Addressing this issue successfully will enrich our understanding of photo-physics in hybrid perovskites and facilitate the experimental search for realistic optoelectronic applications. In this letter, we systematically investigate that the effects of SOC and vibrational motion of the organic cations in $\mathrm{MAPbI}_{3}$ via first-principles calculations as outlined in Methods in the Supporting Information (SI). Our simulations determine the DOS broadening within the VB in cubic, tetragonal and orthorhombic phases at $400,300 \mathrm{~K}$ and $100 \mathrm{~K}$ temperatures, respectively. This phenomenon stems from both the molecular vibrations in the cations and the anharmonicity in the motion of the iodine atoms. The MA cations' vibrations are associated with the moderate $\mathrm{C}-\mathrm{N}$ stretch and $\mathrm{CH}_{3}$ bend, as well as the strong $\mathrm{CH}_{3}$ and $\mathrm{NH}_{3}^{+}$stretches. Knowledge of the specific atomistic motions and interactions that dominate structural and electronic properties is helpful when substituting the organic cation in order to tune materials properties.

Electronic structure calculations for the optimized structures of $\mathrm{MAPbI}_{3}$ (Figure 1) provide a starting point for pinpointing the cause of broadened DOS captured by experimental work. Figure 2 presents the DOS plots with and without SOC for the cubic phase of $\mathrm{MAPbI}_{3}$. These are compared to those of pure inorganic $\mathrm{CsPbI}_{3}$ models taking the same lattice parameters as the corresponding $\mathrm{MAPbI}_{3}$ does. We notice that addition of SOC leads to a shift in energy near the $\mathrm{CB}$ edge producing a reduced band gap, which agrees with previously published electronic structure calculations. ${ }^{29}$ The effect of SOC is not localized to specific momentum values but is known to shift band energies across all sampled k-points in the system. The SOC energy gaps are 0.67 and $0.63 \mathrm{eV}$ for cubic $\mathrm{MAPbI}_{3}$ and the corresponding $\mathrm{CsPbI}_{3}$ model, respectively. These values are significantly corrected by about 0.99 and 0.94 
eV compared to results obtained without SOC. Similar corrections of 0.9 and $1.0 \mathrm{eV}$ are also observed for tetragonal and orthorhombic phases, respectively (Figure S1 and S2), in agreement with previous results. ${ }^{30}$ When focusing on electronic states deeper in the VB, one can see a distinction in how SOC affects the $\mathrm{Cs}$ and $\mathrm{MA}$ systems. For the $\mathrm{CsPbI}_{3}$ system, there exists a Cs peak located near $-8.5 \mathrm{eV}$ with a SOC splitting amounting to $1.53 \mathrm{eV}$ from the Cs p orbitals (Figure 2a and c). From atomic energy level tables, ${ }^{44}$ the +1 oxidation state of a Cs cation has a SOC splitting of about $1.22 \mathrm{eV}$ between the p orbitals of total angular momentum $\mathrm{J}=1 / 2$ and $\mathrm{J}=3 / 2$, which is about $0.31 \mathrm{eV}$ lower than the calculated splitting. In contrast, the energy structure deep within the $\mathrm{VB}$ for the $\mathrm{MAPbI}_{3}$ system, is relatively unchanged with the addition of SOC (Figure 2b), rationalized by a negligible SOC effect from light-elements-constituted MA molecules. Additionally, SOC leads to the subsequent splitting of $\mathrm{Pb}-5 \mathrm{~d}$ orbitals at about $-17 \mathrm{eV}$ into two individual peaks (Figure S3), displaying a SOC splitting of $2.58 \mathrm{eV}$ between the $5 \mathrm{~d}$ orbitals of total angular momentum $J=3 / 2$ and $J=5 / 2$, in nice agreement with $2.65 \mathrm{eV}$ as reported in atomic energy level tables. ${ }^{44}$ This splitting has a minor impact on the electronic structure due to its extremely deep position. In the energy range from -12.5 to $-4 \mathrm{eV}$, there are only a few electronic states associated with $\mathrm{Pb}$ or I atoms (Figure $2 \mathrm{~d}$ ). Moreover, SOC does not produce any sizable enlargement of the total DOS in this energy range down to $-12.5 \mathrm{eV}$ (Figure $2 \mathrm{~b}$ ).

Next, we consider the effect of molecular motion as a possible cause of a broadened DOS within the VB. To this end, we run a series of MD trajectories for Cs and MA-based perovskites adopting NVT thermostat condition. These simulations were performed at temperatures of 400, 300 and $100 \mathrm{~K}$ for the cubic, tetragonal and orthorhombic phases, respectively, on different sizes of periodic cells (see Methods in SI). Since SOC does very little to modify the electronic DOS in the energy range of interest, we neglect SOC in the calculations of MD. The radial pair distribution functions (RDF's) between the different atoms provides a clear picture of the magnitude of the vibrations of the different atoms around their equilibrium positions. As shown in Figures S4-7, the RDF's for the inorganic $\mathrm{PbI}_{3}$ lattice are 
typical for a solid with clearly defined peaks broadened around their optimized positions. However, owing to higher mobility, the situation is different for RDF's involving atoms of the organic cation. There is much more variability for $\mathrm{N}-\mathrm{N}$ pairs in $\mathrm{MAPbI}_{3}$ than $\mathrm{Cs}-\mathrm{Cs}$ pairs in $\mathrm{CsPbI}_{3}$, especially for the high temperature cubic phase. This effect also appreciable in the other two phases, overall being in a good agreement with previous studies. ${ }^{45-47}$

It is expected that the high frequency motions and vibrations of the organic cations could broaden the DOS, especially deep in the VB. We next identify the atomic species participating in the state energy fluctuations so that we can investigate whether the broadened DOS is based on molecular motion or not. While it has been established that SOC has very little effect on the molecular motion and lattice parameters, ${ }^{48}$ in order to test the effect of SOC on the electronic structure we calculated the time-averaged DOS over 100 configurations with SOC along the 15 ps NVT MD trajectories on the $2 \times 2 \times 2$ cubic $\mathrm{MAPbI}_{3}$ cell. As shown in Figure S8, we can find that SOC does not lead to any sizable DOS broadening in this energy range related to the electronic states of $\mathrm{PbI}_{6}$ octahedrons. Consequently, we exclude the SOC effect in forthcoming calculations of massive DOS dynamics, for which the final 400 geometries along each MD trajectory were extracted. Figure 3a demonstrates three broadened DOS peaks (denoted as peak I, II, III) existing deep inside the VB for cubic $\mathrm{MAPbI}_{3}$, located around $-5.0,-9.2$ and $-12.5 \mathrm{eV}$, respectively. Similar peak broadening is also found in the other two lower temperature phases of $\mathrm{MAPbI}_{3}$, differing only by a narrower half-width of three main broadening peaks (Figure $3 \mathrm{~b}$ and $\mathrm{c}$ ). This is due to a reduction in disordered states ${ }^{23,45-47}$ and weakened vibrational motion of the MA cations from temperature effects. Indeed, for $\mathrm{CsPbI}_{3}$ (Figure $\mathrm{S} 9$ ) the time averaged DOS over the entire energy range is almost the same as the ground state of $\mathrm{CsPb}_{3}$, i.e. without significantly broadened DOS peaks as found in the hybrid $\mathrm{MAPbI}_{3}$. The lack of DOS broadening in the Cs system is thus attributed to the absence of MA vibrational modes. As the electronic dimensionality is increased, the electron mobility is enhanced. Although these electrons do not contribute directly to important electronic transitions in devices, they increase the electronic 
dimensionality deep within the VB. ${ }^{19}$

Note that since our MD calculations are based on a volume-fixed ensemble to match the experimental lattice of $\mathrm{MAPbI}_{3}$ in each phase, the fluctuations in the system may not be strictly gauged. As reported in ref., ${ }^{46}$ the MA cations may rotate in NPT conditions that allow volume fluctuations. As soon as the volume restriction is relaxed in the MD simulations, the large distribution of $\mathrm{PbI}_{6}$ octahedral tilting accompanied by an increase in the anharmonicity in the motion of the iodine atoms will occur, which has been observed in $\mathrm{CsPbI}_{3}, \mathrm{MAPbI}_{3}$ and $\mathrm{FAPbI}_{3}$ crystals. ${ }^{46,47}$ It is expected that such anharmonicity in the iodine atom motion could influence the behaviors of DOS broadening. To confirm this point, MD calculations based on the NPT ensemble were carried out on cubic phase $\mathrm{MAPbI}_{3}$ at $450 \mathrm{~K}$, which are based on a $4 \times 4 \times 4$ supercell by replicating the cubic unit cell four times in each Cartesian direction (see ref. ${ }^{47}$ for details). As demonstrated in Figure 3 d there exists an obvious broadening of the electronic states up to $0.5 \mathrm{eV}$ at around $-11.5 \mathrm{eV}$. This confirms that the anharmonicity in the motion of the iodine atoms does contribute to DOS broadening in specific energy regions. Reversely and noteworthy, NVT calculations are not able to reveal any broadening related to the inorganic framework (vide supra). Although the anharmonicity of the I atoms does broaden the DOS, it is a smaller contribution compared to the effect of MA vibrations. Thus, we analyze next the contribution from MA vibrations to the broadening of the DOS.

We have calculated the intra-molecular vibrational modes of the free MA cation. There are 18 molecular vibrational modes, associated with the $\mathrm{C}_{3 v}$ symmetry of the structure, dividing the internal modes into one $A 2$, five symmetric $A 1$ and twelve two-fold degenerate asymmetric $E$ species. ${ }^{49,50}$ The calculated vibrational frequencies of the isolated MA cation at its optimized configuration are listed in Table 1 and are visualized in Figures S10-S11. The six types of vibrational modes in ascending order are $\mathrm{C}-\mathrm{N}$ stretch $\left(281,871 \mathrm{~cm}^{-1}\right)$, $\mathrm{CH}_{3}-\mathrm{NH}_{3}^{+} \operatorname{rock}\left(909,1256 \mathrm{~cm}^{-1}\right), \mathrm{CH}_{3}$ bend $\left(1437,1473 \mathrm{~cm}^{-1}\right), \mathrm{NH}_{3}^{+}$bend $(1545,1679$ $\left.\mathrm{cm}^{-1}\right), \mathrm{CH}_{3}$ stretch $\left(2963,3064 \mathrm{~cm}^{-1}\right)$, and $\mathrm{NH}_{3}^{+}$stretch $\left(3260,3341 \mathrm{~cm}^{-1}\right)$, being in a good 
agreement with previous results. ${ }^{26,49-53}$

Switching to $\mathrm{MAPbI}_{3}$ periodic systems, each $\mathrm{MAPbI}_{3}$ phase manifests similar vibrational properties. The corresponding vibrational spectrum is mainly divided into three energetic regions: (1) the low frequency band from $0-150 \mathrm{~cm}^{-1}$; (2) a mid frequency range from $280-1700 \mathrm{~cm}^{-1}$, and (3) a high-frequency window from $2900-3300 \mathrm{~cm}^{-1} \cdot{ }^{25,26}$ The lowfrequency modes are related entirely to the vibrations of the $\mathrm{PbI}_{6}$ octahedra, while the midand high-frequency modes are linked to the vibrations of MA cations. We computed the Fourier transforms of the state energies in the region of interest, deep within the VB, in order to determine which molecular vibrational modes strongly couple to the electronic degrees of freedom (see Methods in SI). The resulting molecular vibrational spectra for the three crystal phases are shown in Figures 4 and S11-12 for peak I, II and III, respectively, which are in a good agreement with previous experimental values (Table 1). ${ }^{50,53}$ In cubic $\mathrm{MAPbI}_{3}$, the main vibrations contributing to peaks I and III originate from the $\mathrm{CH}_{3} / \mathrm{NH}_{3}^{+}$bend (about 1400 $\mathrm{cm}^{-1}$ ) and $\mathrm{CH}_{3}$ stretch a about $3000 \mathrm{~cm}^{-1}$ (Figure $\mathrm{S} 12-13$ ). The predominant vibrations contributing to peak II are mainly from $\mathrm{CH}_{3}$ bend (about $1400 \mathrm{~cm}^{-1}$ ), $\mathrm{NH}_{3}^{+}$stretch (about $3100 \mathrm{~cm}^{-1}$ ), as well as small amounts of $\mathrm{C}-\mathrm{N}$ stretch (about $950 \mathrm{~cm}^{-1}$ ) and $\mathrm{NH}_{3}^{+}$bend (about $1600 \mathrm{~cm}^{-1}$ ) (Figure 4). Similar results are obtained for the tetragonal and orthorhombic phases, except that the intensities are more attenuated due to the weakened vibrational amplitudes as temperature decreases (Figures S11-12). Exceptions are the broadened peaks I and II which couple to a slightly enhanced vibration along the $\mathrm{C}-\mathrm{N}$ axis as compared to that in the cubic phase. Moreover, the $\mathrm{CH}_{3}$ bends for peak I and III in the orthorhombic phase are almost non-existent due to the temperature effect. The two $\mathrm{CH}_{3}$ stretches (2963, $3064 \mathrm{~cm}^{-1}$ ) for peaks I and III become distinct from each other. Altogether, we mainly attribute widening of DOS deep inside $\mathrm{VB}$ in the three $\mathrm{MAPbI}_{3}$ phases to mostly asymmetric $\mathrm{CH}_{3} / \mathrm{NH}_{3}^{+}$stretches, moderate $\mathrm{CH}_{3}$ bend, and $\mathrm{C}-\mathrm{N}$ stretch. Thus the observed coupling is exclusive to mid-frequency and high-frequency vibrational modes.

In summary, we computationally study the origin of the density of states broadening 
experimentally reported in the hybrid organic-inorganic perovskite $\mathrm{MAPbI}_{3}$, using $\mathrm{CsPbI}_{3}$ as a reference material. We show that spin-orbit coupling mainly modifies the density of states close to the Fermi level and does not contribute to its broadening deep within the valence band. From ab initio molecular dynamics simulations and electronic structure calculations, we further analyze the contribution of thermal vibrations to three phases of $\mathrm{MAPbI}_{3}$. As expected, upon reducing the temperature, the broadening gradually reduces when going from the cubic to tetragonal and to orthorhombic phases, consistent with experimental findings. The density of states broadening is ascribed to two different contributions. For states about $12.5 \mathrm{eV}$ below the top of the valence band, this broadening can be traced back to the anharmonicity in the motion of the iodine atoms. In additional, vibrational modes of the MA cation provide important additional contributions to the density of states broadening in a wide energy range. Contrastingly, SOC does not produce any sizable broadening of the total DOS. Specifically, MA cations' vibrations mainly stem from the C-N stretch (about 950 $\mathrm{cm}^{-1}$ ), $\mathrm{CH}_{3}$ bend (about $1400-1600 \mathrm{~cm}^{-1}$ ) and $\mathrm{CH}_{3}$ and $\mathrm{NH}_{3}^{+}$stretches (from 2950-3300 $\left.\mathrm{cm}^{-1}\right)$. These results present a detailed theoretical perspective on the structural dynamics in hybrid perovskites, rationalize experimental observations, and may guide future studies toward desired functionalities in optoelectronic applications.

\section{Supporting Information Available}

The following files are available free of charge. Methods, Ground state DOS for tetragonal and orthorhombic $\mathrm{CsPbI}_{3}$ and $\mathrm{MAPbI}_{3}$ phases, partial DOS of cubic $\mathrm{MAPbI}_{3}$ with $\mathrm{Pb}-\mathrm{d}$ states included, radial distribution functions in tetragonal and orthorhombic phases, time averaged DOS for $\mathrm{CsPbI}_{3}$ and for $\mathrm{MAPbI}_{3}$ with SOC, calculated vibrational eigenvectors of isolated MA cation and Fourier transforms of the electronic state I and III in three $\mathrm{MAPbI}_{3}$ phases. 


\section{AUTHOR INFORMATION}

\section{Corresponding Author}

*L.Z.: Email: ljzhou86@lanl.gov *S.T: Email: serg@lanl.gov

\section{Notes}

The authors declare no competing financial interest.

\section{Acknowledgement}

The work at Los Alamos National Laboratory (LANL) was supported by the LANL LDRD program (L.Z., A.J.N. and S.T.). This work was done in part at the Center for Nonlinear Studies (CNLS) and the Center for Integrated Nanotechnologies (CINT), a U.S. Department of Energy and Office of Basic Energy Sciences user facility, at LANL. This research used resources provided by the LANL Institutional Computing Program. LANL is operated by Los Alamos National Security, LLC, for the National Nuclear Security Administration of the U.S. Department of Energy under contract DE-AC52-06NA25396. The work in France was supported by Cellule Energie du CNRS (SOLHYBTRANS Project) and University of Rennes 1 (Action Incitative, Défis Scientifique Emergents 2015). 
(a)

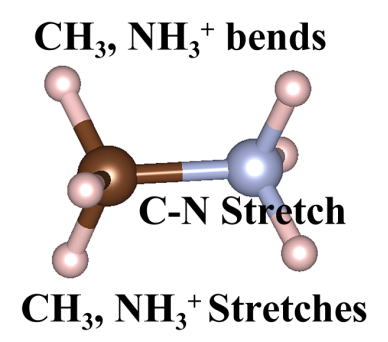

MA

(c)

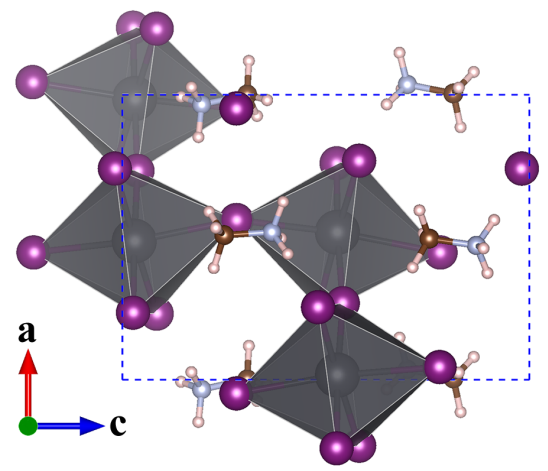

(b)

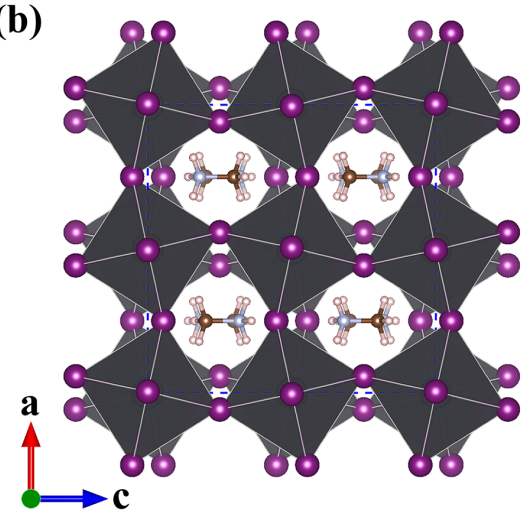

(d)

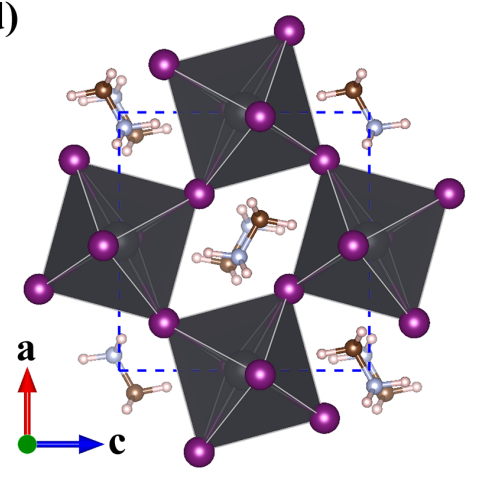

Figure 1: (a): Schematic diagram of the MA molecule. Three main vibrational modes of MA molecule's vibration are marked. (b) $\mathrm{MAPbI}_{3}$ crystal structures for (b) cubic, (c) tetragonal and (d) orthorhombic phases. 
Cubic $\mathrm{CsPbI}_{3}$
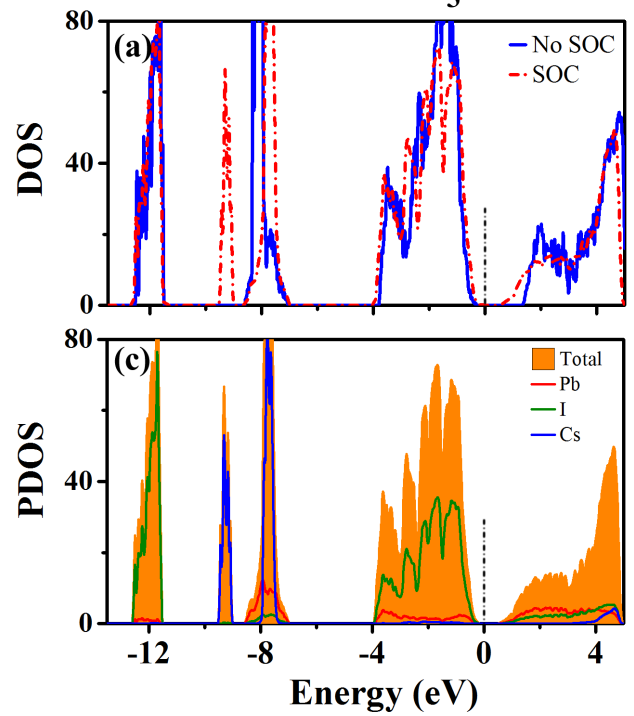

Cubic $\mathrm{MAPbI}_{3}$
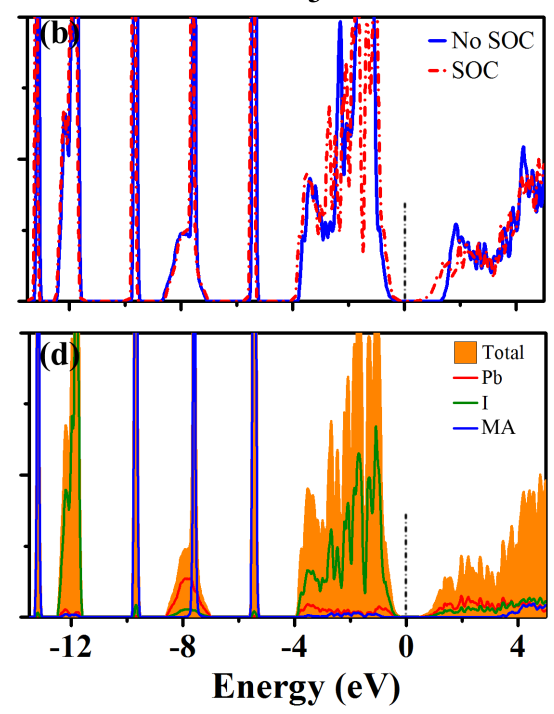

Figure 2: Ground state electronic structure corresponding to the cubic $\mathrm{CsPbI}_{3}$ (a, c) and optimized $\mathrm{MAPbI}_{3}$ (b, d) models. The top panels compare the calculated density of states (DOS) with and without spin-orbit coupling (SOC) as dashed red and solid blue lines, respectively. The bottom panels provide the SOC partial density of states (PDOS) with $\mathrm{Pb}$ (red), I (green), and the Cs or MA cations (blue), respectively. The Fermi energy is set to zero. 

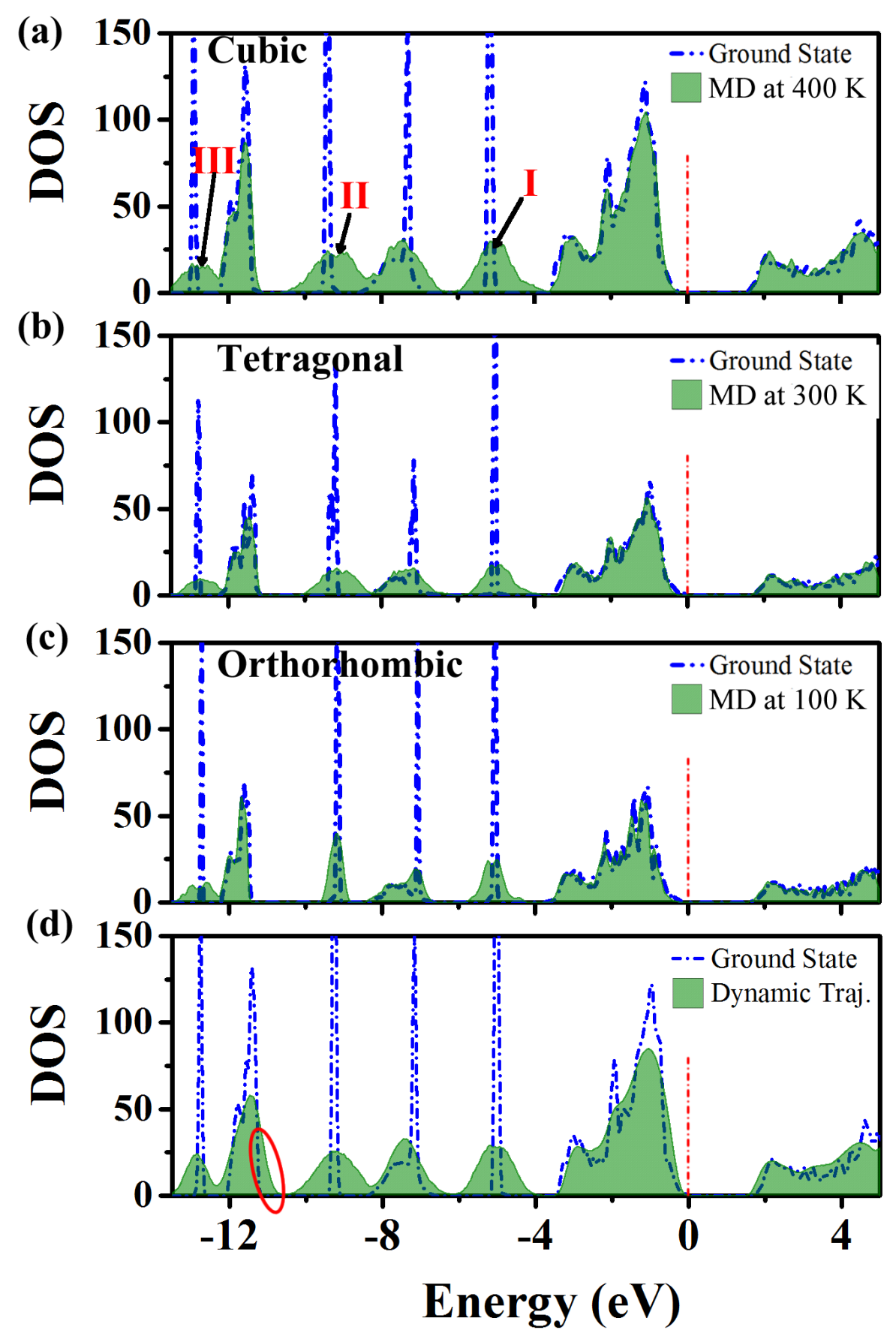

Figure 3: Time averaged DOS of (a) cubic, (b) tetragonal and (c) orthorhombic $\mathrm{MAPbI}_{3}$ along MD trajectories under NVT conditions, overlaid with the ground state DOS. The Fermi levels are set to 0 . The broadened DOS peaks from MA cations are indicated by Roman numbers of I, II and III in panel (a). (d) Time averaged DOS for cubic $\mathrm{MAPbI}_{3}$ along MD trajectories performed under NPT conditions and $4 \times 4 \times 4$ supercell, overlaid with the static DOS. The red circle indicates the contribution from the anharmonicity of I atoms' motion. 

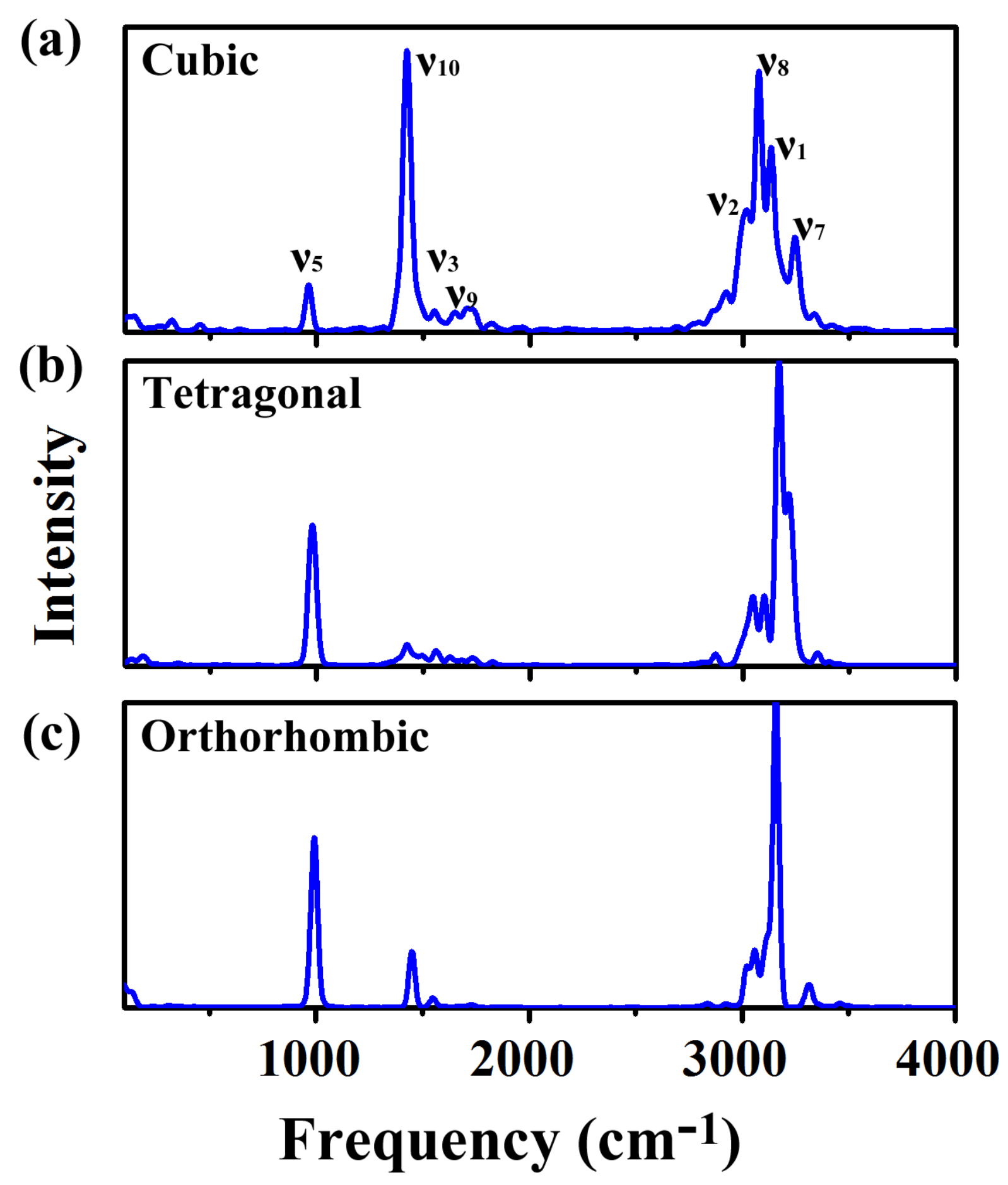

Figure 4: Fourier transforms of the state energies of the electronic states relevant to peak II (Figure 3a) in three phases of $\mathrm{MAPbI}_{3}$ : (a) cubic, (b) tetragonal, (c) orthorhombic. The energy fluctuations are due to molecular vibrational modes of the MA cations. Peak assignments for modes in panel (a) are suggestions only. 
Table 1: Fundamental Vibrations of the Isotopic MA cation Calculated by B3LYP and MP2 Methods and, Experimental Measured Vibrational Modes for $\mathrm{MAPbI}_{3}$ from references ${ }^{50,53}\left(\mathrm{~cm}^{-1}\right)$.

\begin{tabular}{lcccccc}
\hline \multirow{2}{*}{ Mode } & \multirow{2}{*}{ Species } & \multicolumn{2}{c}{ MA } & & \multicolumn{2}{c}{$\mathrm{MAPbI}_{3}$} \\
\cline { 3 - 4 } \cline { 6 - 7 } & & this work & $\mathrm{MP2}^{53}$ & & Ref $^{50}$ & Ref $^{53}$ \\
\hline$v_{12}, \mathrm{CH}_{3}-\mathrm{NH}_{3}^{+}$rock & $E$ & 909 & 1032 & & 903.4 & 911 \\
$v_{5}, \mathrm{C}-\mathrm{N}$ stretch & $A_{1}$ & 871 & 982 & & 990 & 960 \\
$v_{11}, \mathrm{CH}_{3}-\mathrm{NH}_{3}^{+}$rock & $E$ & 1256 & 1409 & & 1248.9 & 1250 \\
$v_{4}$, sym. $\mathrm{CH}_{3}$ bend & $A_{1}$ & 1437 & 1597 & & 1415 & 1385 \\
$v_{10}$, asym. $\mathrm{CH}_{3}$ bend & $E$ & 1473 & 1620 & & 1452.3 & 1423 \\
$v_{3}$, sym. $\mathrm{NH}_{3}^{+}$bend & $A_{1}$ & 1545 & 1691 & & 1469.9 & 1469 \\
$v_{9}$, asym. $\mathrm{NH}_{3}^{+}$bend & $E$ & 1679 & 1809 & & 1581 & 1577 \\
$v_{6}$, torsion & $A_{2}$ & 281 & & & \\
$v_{2}$, sym. $\mathrm{CH}_{3}$ stretch & $A_{1}$ & 2963 & 3164 & & 2896 & 2916 \\
$v_{8}$, asym. $\mathrm{CH}_{3}$ stretch & $E$ & 3064 & 3263 & & 2974.8 & 2958 \\
$v_{1}$, sym. $\mathrm{NH}_{3}^{+}$stretch & $A_{1}$ & 3260 & 3446 & & 3188 & 3132 \\
$v_{7}$, asym. $\mathrm{NH}_{3}^{+}$stretch & $E$ & 3341 & 3519 & & 3225.6 & 3179 \\
\hline
\end{tabular}




\section{References}

(1) Saparov, B.; Mitzi, D. B. OrganicInorganic Perovskites: Structural Versatility for Functional Materials Design. Chem. Rev. 2016, 116, 4558-4596.

(2) Nie, W.; Tsai, H.; Asadpour, R.; Blancon, J.-C.; Neukirch, A. J.; Gupta, G.; Crochet, J. J.; Chhowalla, M.; Tretiak, S.; Alam, M. A. et al. High-efficiency Solutionprocessed Perovskite Solar Cells with Millimeter-scale Grains. Science 2015, 347, 522525.

(3) Kim, Y. C.; Jeon, N. J.; Noh, J. H.; Yang, W. S.; Seo, J.; Yun, J. S.; Ho-Baillie, A.; Huang, S.; Green, M. A.; Seidel, J. et al. Beneficial Effects of $\mathrm{PbI}_{2}$ Incorporated in Organo-Lead Halide Perovskite Solar Cells. Adv. Energy Mater. 2016, 6, 1502104.

(4) Yang, W. S.; Park, B.-W.; Jung, E. H.; Jeon, N. J.; Kim, Y. C.; Lee, D. U.; Shin, S. S.; Seo, J.; Kim, E. K.; Noh, J. H. et al. Iodide management in formamidinium-leadhalide-based perovskite layers for efficient solar cells. Science 2017, 356, 1376-1379.

(5) Srimath Kandada, A. R.; Neutzner, S.; DInnocenzo, V.; Tassone, F.; Gandini, M.; Akkerman, Q. A.; Prato, M.; Manna, L.; Petrozza, A.; Lanzani, G. Nonlinear Carrier Interactions in Lead Halide Perovskites and the Role of Defects. J. Am. Chem. Soc. 2016, 138, 13604-13611.

(6) Eames, C.; Frost, J. M.; Barnes, P. R. F.; ORegan, B. C.; Walsh, A.; Islam, M. S. Ionic Transport in Hybrid Lead Iodide Perovskite Solar Cells. Nat. Commun. 2015, 6, 7497.

(7) Yang, T.-Y.; Gregori, G.; Pellet, N.; Grtzel, M.; Maier, J. The Significance of Ion Conduction in a Hybrid OrganicInorganic Lead-Iodide-Based Perovskite Photosensitizer. Angew. Chem. 2015, 127, 8016-8021.

(8) Zhang, Y.; Liu, M.; Eperon, G. E.; Leijtens, T. C.; McMeekin, D.; Saliba, M.; Zhang, W.; Bastiani, M. d.; Petrozza, A.; Herz, L. M. et al. Charge Selective Con- 
tacts, Mobile Ions and Anomalous Hysteresis in Organic-inorganicPerovskite Solar Cells. Mater. Horiz. 2015, 2, 315-322.

(9) Li, Z.; Xiao, C.; Yang, Y.; Harvey, S. P.; Kim, D. H.; Christians, J. A.; Yang, M.; Schulz, P.; Nanayakkara, S. U.; Jiang, C.-S. et al. Extrinsic Ion Migration in Perovskite Solar Cells. Energy Environ. Sci. 2017, 10, 1234-1242.

(10) Yuan, Y.; Huang, J. Ion Migration in Organometal Trihalide Perovskite and Its Impact on Photovoltaic Efficiency and Stability. Acc. Chem. Res. 2016, 49, 286-293.

(11) Lee, H.; Gaiaschi, S.; Chapon, P.; Marronnier, A.; Lee, H.; Vanel, J.-C.; Tondelier, D.; Boure, J.-E.; Bonnassieux, Y.; Geffroy, B. Direct Experimental Evidence of Halide Ionic Migration under Bias in $\mathrm{CH}_{3} \mathrm{NH}_{3} \mathrm{PbI}_{3-\mathrm{x}} \mathrm{Cl}_{\mathrm{x}}$-Based Perovskite Solar Cells Using GD-OES Analysis. ACS Energy Lett. 2017, 2, 943-949.

(12) Neukirch, A. J.; Nie, W.; Blancon, J.-C.; Appavoo, K.; Tsai, H.; Sfeir, M. Y.; Katan, C.; Pedesseau, L.; Even, J.; Crochet, J. J. et al. Polaron Stabilization by Cooperative Lattice Distortion and Cation Rotations in Hybrid Perovskite Materials. Nano Lett. 2016, 16, 3809-3816.

(13) Nie, W.; Blancon, J.-C.; Neukirch, A. J.; Appavoo, K.; Tsai, H.; Chhowalla, M.; Alam, M. A.; Sfeir, M. Y.; Katan, C.; Even, J. et al. Light-activated Photocurrent Degradation and Self-healing in Perovskite Solar Cells. Nat. Commun. 2016, 7, 11574.

(14) Miyata, K.; Meggiolaro, D.; Trinh, M. T.; Joshi, P. P.; Mosconi, E.; Jones, S. C.; Angelis, F. D.; Zhu, X.-Y. Large Polarons in Lead Halide Perovskites. Sci. Adv. 2017, 3, e1701217.

(15) Zhu, X.-Y.; Podzorov, V. Charge Carriers in Hybrid OrganicInorganic Lead Halide Perovskites Might Be Protected as Large Polarons. J. Phys. Chem. Lett. 2015, 6, $4758-4761$. 
(16) Frost, J. M.; Butler, K. T.; Brivio, F.; Hendon, C. H.; van Schilfgaarde, M.; Walsh, A. Atomistic Origins of High-Performance in Hybrid Halide Perovskite Solar Cells. Nano Lett. 2014, 14, 2584-2590.

(17) Liu, S.; Zheng, F.; Koocher, N. Z.; Takenaka, H.; Wang, F.; Rappe, A. M. Ferroelectric Domain Wall Induced Band Gap Reduction and Charge Separation in Organometal Halide Perovskites. J. Phys. Chem. Lett. 2015, 6, 693-699.

(18) Kutes, Y.; Ye, L.; Zhou, Y.; Pang, S.; Huey, B. D.; Padture, N. P. Direct Observation of Ferroelectric Domains in Solution-Processed $\mathrm{CH}_{3} \mathrm{NH}_{3} \mathrm{PbI}_{3}$ Perovskite Thin Films. J. Phys. Chem. Lett. 2014, 5, 3335-3339.

(19) Xiao, Z.; Meng, W.; Wang, J.; Mitzi, D. B.; Yan, Y. Searching for Promising New Perovskite-based Photovoltaic Absorbers: the Importance of Electroni Dimensionality. Mater. Horiz. 2017, 4, 206-216.

(20) Motta, C.; El-Mellouhi, F.; Kais, S.; Tabet, N.; Alharbi, F.; Sanvito, S. Revealing the Role of Organic Cations in Hybrid Halide Perovskite $\mathrm{CH}_{3} \mathrm{NH}_{3} \mathrm{PbI}_{3}$. Nat. Commun. 2015, 6, 7026 .

(21) Miyata, A.; Mitioglu, A.; Plochocka, P.; Portugall, O.; Wang, J. T.-W.; Stranks, S. D.; Snaith, H. J.; Nicholas, R. J. Direct Measurement of the Exciton Binding Energy and Effective Masses for Charge Carriers in Organic-inorganic Tri-halide Perovskites. Nat. Phys. 2015, 11, 582-587.

(22) Kato, M.; Fujiseki, T.; Miyadera, T.; Sugita, T.; Fujimoto, S.; Tamakoshi, M.; Chikamatsu, M.; Fujiwara, H. Universal Rules for Visible-light Absorption in Hybrid Perovskite Materials. J. Appl. Phys. 2017, 121, 115501.

(23) Kawamura, Y.; Mashiyama, H.; Hasebe, K. Structural Study on CubicTetragonal Transition of $\mathrm{CH}_{3} \mathrm{NH}_{3} \mathrm{PbI}_{3}$. J. Phys. Soc. Jpn. 2002, 71, 1694-1697. 
(24) Ma, J.; Wang, L.-W. The Nature of Electron Mobility in Hybrid Perovskite $\mathrm{CH}_{3} \mathrm{NH}_{3} \mathrm{PbI}_{3}$. Nano Lett. 2017, 17, 3646-3654.

(25) Bakulin, A. A.; Selig, O.; Bakker, H. J.; Rezus, Y. L.; Mller, C.; Glaser, T.; Lovrincic, R.; Sun, Z.; Chen, Z.; Walsh, A. et al. Real-Time Observation of Organic Cation Reorientation in Methylammonium Lead Iodide Perovskites. J. Phys. Chem. Lett. 2015, $6,3663-3669$.

(26) Brivio, F.; Frost, J. M.; Skelton, J. M.; Jackson, A. J.; Weber, O. J.; Weller, M. T.; Goi, A. R.; Leguy, A. M. A.; Barnes, P. R. F.; Walsh, A. Lattice Dynamics and Vibrational Spectra of the Orthorhombic, Tetragonal, and Cubic Phases of Methylammonium Lead Iodide. Phys. Rev. B 2015, 92, 144308.

(27) Lindblad, R.; Bi, D.; Park, B.-w.; Oscarsson, J.; Gorgoi, M.; Siegbahn, H.; Odelius, M.; Johansson, E. M. J.; Rensmo, H. Electronic Structure of $\mathrm{TiO}_{2} / \mathrm{CH}_{3} \mathrm{NH}_{3} \mathrm{PbI}_{3}$ Perovskite Solar Cell Interfaces. J. Phys. Chem. Lett. 2014, 5, 648-653.

(28) Miller, E. M.; Zhao, Y.; Mercado, C. C.; Saha, S. K.; Luther, J. M.; Zhu, K.; Stevanovi, V.; Perkins, C. L.; Lagemaat, J. v. d. Substrate-controlled Band Positions in $\mathrm{CH}_{3} \mathrm{NH}_{3} \mathrm{PbI}_{3}$ Perovskite Films. Phys. Chem. Chem. Phys. 2014, 16, 22122-22130.

(29) Even, J.; Pedesseau, L.; Jancu, J.-M.; Katan, C. Importance of Spin-Orbit Coupling in Hybrid Organic/Inorganic Perovskites for Photovoltaic Applications. J. Phys. Chem. Lett. 2013, 4, 2999-3005.

(30) Katan, C.; Pedesseau, L.; Kepenekian, M.; Rolland, A.; Even, J. Interplay of Spinorbit Coupling and Lattice Distortion in Metal Substituted 3D Tri-chloride Hybrid perovskites. J. Mater. Chem. A 2015, 3, 9232-9240.

(31) Motoki, K.; Miyazawa, Y.; Kobayashi, D.; Ikegami, M.; Miyasaka, T.; Yamamoto, T.; Hirose, K. Degradation of $\mathrm{CH}_{3} \mathrm{NH}_{3} \mathrm{PbI}_{3}$ Perovskite Due to Soft x-ray Irradiation as An- 
alyzed by an X-ray Photoelectron spectroscopy time-dependent measurement method. J. Appl. Phys. 2017, 121, 085501.

(32) Lindblad, R.; Jena, N. K.; Philippe, B.; Oscarsson, J.; Bi, D.; Lindblad, A.; Mandal, S.; Pal, B.; Sarma, D. D.; Karis, O. et al. Electronic Structure of CH3NH3PbX3 Perovskites: Dependence on the Halide Moiety. J. Phys. Chem. C 2015, 119, 18181825.

(33) Endres, J.; Egger, D. A.; Kulbak, M.; Kerner, R. A.; Zhao, L.; Silver, S. H.; Hodes, G.; Rand, B. P.; Cahen, D.; Kronik, L. et al. Valence and Conduction Band Densities of States of Metal Halide Perovskites: A Combined ExperimentalTheoretical Study. J. Phys. Chem. Lett. 2016, 7, 2722-2729.

(34) Wei, H.; Fang, Y.; Mulligan, P.; Chuirazzi, W.; Fang, H.-H.; Wang, C.; Ecker, B. R.; Gao, Y.; Loi, M. A.; Cao, L. et al. Sensitive X-ray Detectors Made of Methylammonium Lead Tribromide Perovskite Single Crystals. Nat. Photon. 2016, 10, 333-339.

(35) Yakunin, S.; Protesescu, L.; Krieg, F.; Bodnarchuk, M. I.; Nedelcu, G.; Humer, M.; Luca, G. D.; Fiebig, M.; Heiss, W.; Kovalenko, M. V. Low-threshold Amplified Spontaneous Emission and Lasing from Colloidal Nanocrystals of Caesium Lead Halide Perovskites. Nat. Commun. 2015, 6, 8056.

(36) Yakunin, S.; Sytnyk, M.; Kriegner, D.; Shrestha, S.; Richter, M.; Matt, G. J.; Azimi, H.; Brabec, C. J.; Stangl, J.; Kovalenko, M. V. et al. Detection of X-ray Photons by Solution-processed Lead Halide Perovskites. Nat. Photon. 2015, 9, 444-449.

(37) Lee, M.-I.; Barragn, A.; Nair, M. N.; Jacques, V. L. R.; Bolloch, D. L.; Fertey, P.; Khaoula Jemli,; Lde, F.; Tripp-Allard, G.; Deleporte, E. et al. First Determination of the Valence Band Dispersion of $\mathrm{CH}_{3} \mathrm{NH}_{3} \mathrm{PbI}_{3}$ Hybrid Organicinorganic Perovskite. J. Phys. D: Appl. Phys. 2017, 50, 26LT02. 
(38) Knutson, J. L.; Martin, J. D.; Mitzi, D. B. Tuning the Band Gap in Hybrid Tin Iodide Perovskite Semiconductors Using Structural Templating. Inorg. Chem. 2005, 44, 46994705.

(39) Bernasconi, A.; Malavasi, L. Direct Evidence of Permanent Octahedra Distortion in $\mathrm{MAPbBr}_{3}$ Hybrid Perovskite. ACS Energy Lett. 2017, 2, 863-868.

(40) Prasanna, R.; Gold-Parker, A.; Leijtens, T.; Conings, B.; Babayigit, A.; Boyen, H.G.; Toney, M. F.; McGehee, M. D. Band Gap Tuning via Lattice Contraction and Octahedral Tilting in Perovskite Materials for Photovoltaics. J. Am. Chem. Soc. 2017, 139, 11117-11124.

(41) Lee, J.-H.; Bristowe, N. C.; Lee, J. H.; Lee, S.-H.; Bristowe, P. D.; Cheetham, A. K.; Jang, H. M. Resolving the Physical Origin of Octahedral Tilting in Halide Perovskites. Chem. Mater. 2016, 28, 4259-4266.

(42) Even, J.; Pedesseau, L.; Jancu, J.-M.; Katan, C. DFT and k.p Modelling of the Phase Transitions of Lead and Tin Halide Perovskites for Photovoltaic Cells. Phys. Status Solidi Rapid Res. Lett. 2014, 8, 31-35.

(43) Berdiyorov, G. R.; Kachmar, A.; El-Mellouhi, F.; Carignano, M. A.; El-Amine Madjet, M. Role of Cations on the Electronic Transport and Optical Properties of LeadIodide Perovskites. J. Phys. Chem. C 2016, 120, 16259-16270.

(44) Kramida, A., Ralchenko, Yu., Reader, J. and NIST ASD Team (2018). NIST Atomic Spectra Database (version 5.5.2), https://physics.nist.gov/asd. National Institute of Standards and Technology, Gaithersburg, MD.

(45) Roiland, C.; Tripp-Allard, G.; Jemli, K.; Alonso, B.; Ameline, J.-C.; Gautier, R.; Bataille, T.; Polls, L. L.; Deleporte, E.; Even, J. et al. Multinuclear NMR as a Tool for Studying Local Order and Dynamics in $\mathrm{CH}_{3} \mathrm{NH}_{3} \mathrm{PbX}_{3}(\mathrm{X}=\mathrm{Cl}, \mathrm{Br}, \mathrm{I})$ Hybrid Perovskites. Phys. Chem. Chem. Phys. 2016, 18, 27133-27142. 
(46) Carignano, M. A.; Saeed, Y.; Aravindh, S. A.; Roqan, I. S.; Even, J.; Katan, C. A Close Examination of the Structure and Dynamics of $\mathrm{HC}\left(\mathrm{NH}_{2}\right)_{2} \mathrm{PbI}_{3}$ by $\mathrm{MD}$ Simulations and Group theory. Phys. Chem. Chem. Phys. 2016, 18, 27109-27118.

(47) Carignano, M. A.; Aravindh, S. A.; Roqan, I. S.; Even, J.; Katan, C. Critical Fluctuations and Anharmonicity in Lead Iodide Perovskites from Molecular Dynamics Supercell Simulations. J. Phys. Chem. C 2017, 121, 20729-20738.

(48) Egger, D. A.; Kronik, L. Role of Dispersive Interactions in Determining Structural Properties of OrganicInorganic Halide Perovskites: Insights from First-Principles Calculations. J. Phys. Chem. Lett. 2014, 5, 2728-2733.

(49) Onoda-Yamamuro, N.; Matsuo, T.; Suga, H. Calorimetric and IR Spectroscopic Studies of Phase Transitions in Methylammonium Trihalogenoplumbates (II)+. J. Phys. Chem. Solids 1990, 51, 1383-1395.

(50) Oxton, I. A.; Knop, O.; Duncan, J. I. The Infrared Spectrum and Force Field of the Methyl-ammonium Ion in $\left(\mathrm{CH}_{3} \mathrm{NH}_{3}\right)_{2} \mathrm{PtCl}_{6}$. J. Mol. Struct. 1977, 38, 25-32.

(51) Quarti, C.; Grancini, G.; Mosconi, E.; Bruno, P.; Ball, J. M.; Lee, M. M.; Snaith, H. J.; Petrozza, A.; Angelis, F. D. The Raman Spectrum of the $\mathrm{CH}_{3} \mathrm{NH}_{3} \mathrm{PbI}_{3}$ Hybrid Perovskite: Interplay of Theory and Experiment. J. Phys. Chem. Lett. 2014, 5, 279-284.

(52) DeFrees, D. J.; McLean, A. D. Molecular Orbital Predictions of the Vibrational Frequencies of Some Molecular Ions. J. Chem. Phys. 1985, 82, 333-341.

(53) Glaser, T.; Mller, C.; Sendner, M.; Krekeler, C.; Semonin, O. E.; Hull, T. D.; Yaffe, O.; Owen, J. S.; Kowalsky, W.; Pucci, A. et al. Infrared Spectroscopic Study of Vibrational Modes in Methylammonium Lead Halide Perovskites. J. Phys. Chem. Lett. 2015, 6, 2913-2918. 